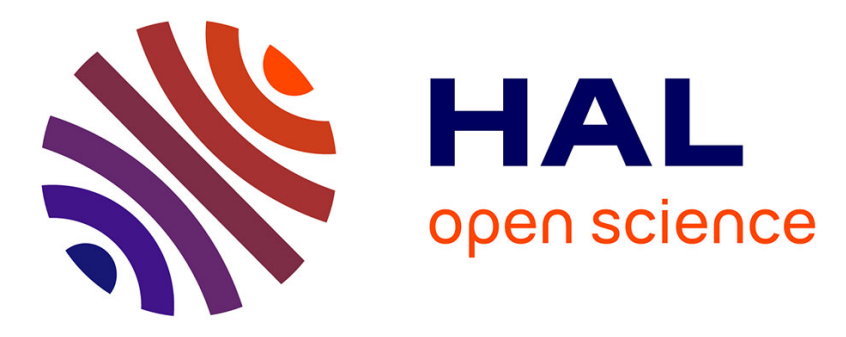

\title{
Determination of resistivity profiles in anti-corrosion coatings from constant-phase-element parameters
}

Marco Musiani, Mark E. Orazem, Nadine Pébère, Bernard Tribollet, Vincent

Vivier

\section{- To cite this version:}

Marco Musiani, Mark E. Orazem, Nadine Pébère, Bernard Tribollet, Vincent Vivier. Determination of resistivity profiles in anti-corrosion coatings from constant-phase-element parameters. Progress in Organic Coatings, 2014, 77 (12), pp.2076-2083. 10.1016/j.porgcoat.2013.12.013 . hal-01082857

\section{HAL Id: hal-01082857 \\ https://hal.sorbonne-universite.fr/hal-01082857}

Submitted on 18 Nov 2014

HAL is a multi-disciplinary open access archive for the deposit and dissemination of scientific research documents, whether they are published or not. The documents may come from teaching and research institutions in France or abroad, or from public or private research centers.
L'archive ouverte pluridisciplinaire $\mathbf{H A L}$, est destinée au dépôt et à la diffusion de documents scientifiques de niveau recherche, publiés ou non, émanant des établissements d'enseignement et de recherche français ou étrangers, des laboratoires publics ou privés. 


\title{
Determination of Resistivity Profiles in Anti-Corrosion Coatings from Constant-Phase-Element
}

\section{Parameters}

\author{
M. Musiani ${ }^{a}$, M. E. Orazem ${ }^{b}$, N. Pébère ${ }^{c, *}$, B. Tribollet $^{d}$, V. Vivier $^{d}$ \\ ${ }^{a}$ Istituto per I'Energetica e le Interfasi, Consiglio Nazionale delle Ricerche,35127 Padova, Italy \\ ${ }^{b}$ Department of Chemical Engineering, University of Florida, Florida 32611, USA \\ ' Université de Toulouse, CIRIMAT, UPS / INPT / CNRS, ENSIACET, 4, allée Emile Monso-BP 44362, 31030 \\ Toulouse cedex 4, France \\ d Laboratoire Interfaces et Systèmes Electrochimiques, UPR 15 du CNRS, Université Pierre et Marie Curie, 75252, \\ Paris cedex 05, France
}

\section{Abstract}

A recently proposed model which explains the CPE response exhibited by metal/coating/electrolyte systems as the result of power-law dependences of the coating resistivity and permittivity along their thickness is revisited and applied to three different coating/electrolyte combinations. It is shown that resistivity profiles may be determined, and their evolution with the time of exposure may be followed. The effect of the assumptions made on coating and electrolyte resistivity on the regressed parameters is discussed and the factors which affect the reliability of the measured water uptake are highlighted. It is shown that both the composition of the coating and that of the test solutions influence the time dependence of the coating performance.

Keywords: CPE; electrolyte uptake; impedance modeling; pores.

* Corresponding author.

Phone: 0033534323423

Fax: 0033534323499

E-mail: Nadine.Pebere@ensiacet.fr (Nadine Pébère) 


\section{Introduction}

Electrochemical impedance spectroscopy is a popular technique for the assessment of the corrosion protection performance of organic coatings. The analysis of impedance data is often based on the equivalent circuit proposed by Beaunier et al. [1] in which the coating capacity and the double layer capacity are replaced by constant phase elements (CPE), the impedance of which is $Z=1 /(j \omega)^{\alpha} Q$. Substitution of CPEs for capacities may strongly improve the quality of the fitting between the experimental data and the equivalent circuit, but creates ambiguities in the physical interpretation of the results, since $Q$ cannot be simply identified with the coating capacity [2] and cannot be directly used to calculate the water uptake in the coating through the Brasher and Kingsbury [3] approach. Various formulas have been proposed for the calculation of the effective capacity from CPE parameters [4,5], but their application requires knowledge of the physical origin of the CPE behavior $[2,6]$.

In a recent paper [7], our group has shown that the CPE behavior observed in the impedance of a coated system may be explained by an inhomogeneous electrolyte uptake if (i) the electrolyte volume fraction decreases from the coating/electrolyte interface to the metal/coating interface according to a power-law and (ii) the resistivity and permittivity of the system are calculated using effective medium theory formulas corresponding to a parallel combination of coating material and electrolyte. This model, henceforth briefly called "power-law model" was compared to experimental data obtained with a hybrid sol-gel coating exposed to a $0.5 \mathrm{M} \mathrm{NaCl}$ solution. It was concluded that, as a result of the progressive electrolyte uptake for increasing exposure time, the resistivity of the coating markedly decreased (after one week, the decrease at the coating/electrolyte interface attained ca. 6 orders of magnitude, with respect to the dry coating), while its permittivity remained practically unchanged. Furthermore, it was shown that the number and/or section of the through pores, i.e. pores as deep as the whole coating thickness, directly connecting the electrolyte with the metal underneath the coating through a low-resistance path, increased with exposure time. These results allowed the extension of a previously derived model [8,9] applied so far to passive oxides and human skin [10], to anti-corrosion coatings.

The present paper aims at extending the investigation described in [7], focusing mainly on three issues: (i) the effect of the assumptions made on coating and electrolyte resistivity on the regressed parameters; (ii) the analysis of the relative amounts of electrolyte within through and short pores; (iii) the analysis of experimental 
data relevant to other coating/electrolyte combinations, based on the power-law model, and a discussion of the different behavior of different systems.

\section{Experimental}

A description of the coatings, their method of preparation, and the electrochemical techniques used for their characterization are presented in this section.

\subsection{Hybrid sol-gel coatings}

Two coatings were prepared. For both systems, the organosiloxane sol was prepared by using 3glycidoxypropylethoxysilane (GLYEO), tetraethylorthosilicate (TEOS) and butanol. A first solution was made by adding a polyaminoamide (PAA) to the sol, with a GLYEO/TEOS/PAA mass ratio of 3:1:2. The solution was prepared with an excess of organosilane, therefore the resulting coating was called $O$. The process takes advantage of the two moieties of the organosilane molecule (GLYEO). The organic part of the GLYEO reacts with the amine functions of the PAA to develop the organic part of the hybrid coating, while the silane part undergoes the usual hydrolysis and condensation steps of sol-gel synthesis, to form a silicon-based network. TEOS was added to increase the coating density. In the second solution, an epoxy compound was added to the first solution, with a GLYEO/TEOS/PAA/Epoxy ratio of 1:1:10:8. Due to the presence of an epoxy in the solution, the resulting coating was called sample E. The addition of the epoxy compound in the liquid sol-gel resin required a different procedure for mixing together the compounds. For this reason, sample $E$ is not comparable, from a chemical point of view, with sample $\mathrm{O}$. The sol-gel films did not contain any pigments or fillers.

The coatings were deposited onto a 2024 T3 aluminium alloy currently used in the aerospace industry. The specimens consisted of $125 \mathrm{~mm} \times 80 \mathrm{~mm} \times 1.6 \mathrm{~mm}$ plates machined from rolled plate. Before painting, the samples were degreased at $60^{\circ} \mathrm{C}(\mathrm{pH}=9)$ for $15 \mathrm{~min}$, rinsed twice with distilled water, then etched in an acid bath at $52{ }^{\circ} \mathrm{C}$ for $10 \mathrm{~min}$, and rinsed again with distilled water. The liquid paints were applied by air spraying and cured at $100^{\circ} \mathrm{C}$ for $1 \mathrm{~h}$. The coatings thickness was measured at several locations from the observation of the cross-sections of the samples by SEM and found to have an average value of $20 \pm 2 \mu \mathrm{m}$.

\subsection{Electrochemical impedance measurements}


A three-electrode electrochemical cell was used in which the coated specimen served as the working electrode.

A cylindrical Plexiglas tube was assembled on top of the coated sample, exposing a surface area of $24 \mathrm{~cm}^{2}$. A large platinum sheet was used as counter-electrode. A saturated mercury/mercurous sulphate reference electrode was used for electrolytes containing $\mathrm{Na}_{2} \mathrm{SO}_{4}$, and a saturated calomel reference electrode was used in electrolytes containing $\mathrm{NaCl}$. The electrochemical cell was open to air and was kept at room temperature with an average value of $17^{\circ} \mathrm{C}$ and which may have undergone fluctuations by $\pm 2^{\circ} \mathrm{C}$. Following our previous work, the resulting variations in the dielectric constant of water were unlikely to have significant effects on the experimental impedance response [9]. Electrochemical impedance measurements were carried out using a Biologic VSP apparatus. The impedance diagrams were obtained under potentiostatic conditions at the corrosion potential over a frequency range of $60 \mathrm{kHz}$ to $10 \mathrm{mHz}$ with 6 points per decade, using a $20 \mathrm{mV}$ peakto-peak sinusoidal voltage. The electrochemical behaviour of the coatings was characterized in either $0.5 \mathrm{M}$ $\mathrm{Na}_{2} \mathrm{SO}_{4}$ or $0.5 \mathrm{M} \mathrm{NaCl}$, for exposure times ranging from $2 \mathrm{~h}$ to $168 \mathrm{~h}$. The $\mathrm{Na}_{2} \mathrm{SO}_{4}$ solution was used to achieve a better knowledge of the intrinsic properties of the coatings because the evolution of the system was slower than in $\mathrm{NaCl}$ solution. The results concerning sample $\mathrm{E}$ in the $\mathrm{NaCl}$ solution were previously reported in [7].

\section{Model for data analysis}

The model employed for the data analysis has been previously described [7]; only a brief summary is reported here. A scheme of a substrate/oxide/coating/electrolyte system, and its equivalent circuit, are presented in Fig.

1. The impedance is assumed to consist of three components connected in series:

i. the uncompensated electrolyte resistance;

ii. the impedance of the coating, discussed below;

iii. the impedance of the oxide covering the Al 2024 alloy; this impedance is accounted for by a constant phase element (CPE) and is no longer discussed in the following.

The coating is assumed to be penetrated by the electrolyte which fills its pores. These pores are represented in Fig. 1 as being straight, although they may be tortuous and have variable lateral dimension. All pores have a mouth at the coating/electrolyte interface, but only a few (henceforth called "through pores") are as deep as the coating and reach the coating/oxide interface, providing a low-resistivity path, with resistance $R_{\text {pore, }}$ between the electrolyte and the oxide. The other pores (henceforth called "short pores") are less deep than the coating thickness $\delta$, and thus different planes parallel to the interfaces, located within the coating at different positions $\xi=x / \delta$, cross different numbers of pores (see sketch in Fig. 2), and different elemental layers 
of the coating, $\mathrm{d} \xi$ thick, have different local electrolyte volume fractions $\phi(\xi)$. Such a representation of the electrolyte-penetrated coating is in agreement with the one proposed by Mišković-Stanković et al. [11,12] on the basis of optical microscopy coupled with image analysis.

The electrolyte volume fraction affects the local resistivity and permittivity of the coating, which are expressed through effective medium theory formulas as

$$
\rho(\xi)^{-1}=\rho_{\mathrm{w}}^{-1} \phi(\xi)+\rho_{\mathrm{c}}^{-1}[1-\phi(\xi)]
$$

and

$$
\varepsilon(\xi)=\varepsilon_{\mathrm{w}} \phi(\xi)+\varepsilon_{\mathrm{c}}[1-\phi(\xi)]
$$

where the subscripts $\mathrm{w}$ and $\mathrm{c}$ refer to the electrolyte and the coating material, respectively. Equations (1) and (2) take into account that, in each elemental layer parallel to the interfaces, each position is occupied by either the coating material or the electrolyte, and so these two media are in parallel to each other. In this approximation it is unimportant whether $\phi(\xi)$ is the result of the presence of a large number of smaller pores or a small number of larger pores. However, $\phi(\xi)$ does not take into account the water present within the coating as individual molecules interacting with polymeric network and not forming aggregates big enough to allow dissolution of salts and generate a conductive medium. Such polymer-bounded water has been reported to be a very significant fraction of the total water uptaken by epoxy coatings [11] and to cause strong plasticization of an epoxy/polyamidoamine coating [13]. Therefore, the water uptake determined through the analysis of experimental data by comparison with the model proposed in [7] is likely to be underestimated. In a following section (see 4.1.3), it will be discussed how a lower resistivity of the electrolyte within the pores, as compared to that of the external solution, may be a further cause for underestimating the water uptake. Under assumption that each elemental layer may be represented by a parallel combination of a resistance $\rho(\xi) \mathrm{d} \xi$ and a capacitance $\varepsilon(\xi) \varepsilon_{0} / \mathrm{d} \xi$, the impedance of the electrolyte-penetrated coating $\left(Z_{\mathrm{c}}\right)$ is calculated as

$$
Z_{\mathrm{c}}(\omega)=\delta \int_{0}^{1} \frac{1}{\rho(\xi)^{-1}+\mathrm{j} \omega \varepsilon(\xi) \varepsilon_{0}} \mathrm{~d} \xi
$$

The contribution of pores that extend to the oxide surface is included separately from the development of equation (3). The overall impedance $Z(\omega)$ of the coating is thereby given by the pore resistance $R_{\text {pore }}$ in parallel with $Z_{\mathrm{c}}$, i.e.

$$
Z(\omega)=\left[Z_{\mathrm{c}}(\omega)^{-1}+R_{\mathrm{pore}}^{-1}\right]^{-1}
$$

To account for the progressive variation in the number of pores along the coating thickness, the hypothesis is made that $\phi(\xi)$ changes from $\phi(0)=0$ at $\xi=0$ to $\phi(\delta)$ at $\xi=1$ according to a power-law, i.e., 


$$
\phi(\xi)=\frac{\phi(\delta) \xi^{\gamma}}{1+\phi(\delta)\left(\xi^{\gamma}-1\right)}
$$

The coating part corresponding to the impedance $Z_{\mathrm{c}}$ contains only "short pores", meaning that no electrolyte reaches the interface between the coating and the oxide and, as a consequence, $\phi(0)=0$.

In reference [7], calculated impedance plots were presented with $\phi(\delta)$ and $R_{\text {pore }}$ as parameters. It was shown that, according to a more general theory reported in [8], $Z(\omega)$ corresponded to a CPE behavior, with a CPE exponent $\alpha$ linked to the power-law exponent $\gamma$ by the equation

$$
\alpha=\frac{\gamma-1}{\gamma}
$$

It was shown in [7] that fitting the above described model to experimental data allowed determination of $\gamma$, $\phi(\delta)$, and $R_{\text {pore, }}$ by assuming known or reasonable values for $\rho_{\mathrm{w}}, \rho_{\mathrm{c}}, \varepsilon_{\mathrm{w}}$ and $\varepsilon_{\mathrm{c}}$. The $\phi(\xi)-\xi$ profiles were calculated by entering the fitted $\gamma$ and $\phi(\delta)$ values into equation (5); hence, $\rho(\xi)$ - $\xi$ profiles corresponding to different immersion times were obtained through equation (1). These profiles, presented in [7], neglected the through pores, and, therefore, at all immersion times, $\rho(0)$ was identical to $\rho_{\mathrm{c}}$. In the present paper, the electrolyte content in the through pores is calculated as

$$
\varphi_{\text {pore }}=\frac{V_{\text {pore }}}{V_{\text {film }}}=\frac{A_{\text {pore }}}{A_{\text {film }}}=\frac{\delta \rho_{\mathrm{w}}}{R_{\text {pore }} A_{\text {film }}}
$$

where $V$ denotes a volume, and $A$ is a surface area (with $R_{\text {pore }}$ normalized to unit surface area and $A_{\text {film }}=1 \mathrm{~cm}^{2}$ ). This allows estimation of the fraction of the uptaken electrolyte that is contained in the through pores and calculation of $\rho(\xi)-\xi$ profiles, taking into account all pores. Owing to the assumed cylindrical geometry of the through pores, $R_{\text {pore }}$ is not a function of position $\xi$.

The above development retains the meaning of $\rho(0)$ as the resistance of the dry coating, $\rho_{\mathrm{c}}$. As may be seen in the subsequent discussion of experimental results, the power-law model does not account adequately for the high-frequency response. The origin of this discrepancy is, as yet, unknown. A tentative explanation might invoke capacitive interactions between adjacent pores. The regression analysis was therefore performed for frequencies up to an upper limit which, for different systems, varied from 0.5 to $5 \mathrm{kHz}$ (see Figures 3 and 6).

\section{Results and Discussion}


The results are presented in terms of the influence of assumed values for parameters $\rho_{w}, \rho_{c}, \varepsilon_{w}$ and $\varepsilon_{c}$ on the fitted parameters, the relative amounts of electrolyte within through and short pores, and the influence of coating formulation and electrolyte composition.

\subsection{Effect of $\rho_{w}, \rho_{c}, \varepsilon_{w}$ and $\varepsilon_{c}$ on fitted parameters}

Coated samples were prepared, and impedance measurements were performed as described in the experimental section. For each combination of coating/electrolytic solution/immersion time the experimental data were compared with the model shown in Fig. 1, using a non-commercial software developed at the LISE CNRS, Paris. This software allowed regression of the model consisting of a combination of passive circuit elements, like $R_{\text {pore }}$, the electrolyte resistance and the CPE parameters relevant to the oxide film, and the analytical expression of $Z_{c}(\omega)$, but did not provide confidence intervals for the best fitted parameters. In our previous work [7], it was explained that, owing to the quasi-capacitive blocking behavior observed at low frequency, the oxide film resistance could be assumed to be very large and therefore its inclusion in the equivalent circuit, in parallel with the $\mathrm{CPE}$, was not necessary. Equally unnecessary were circuit elements accounting for the corrosion reactions, because no sign of corrosion was observed for the immersion times explored in the present investigation.

In the regression procedure, $\gamma, \phi(\delta)$ and $R_{\text {pore }}$ were the only adjustable parameters for the coating; whereas, $\rho_{\mathrm{w}}$, $\rho_{\mathrm{c}}, \varepsilon_{\mathrm{w}}$ and $\varepsilon_{\mathrm{c}}$ were given fixed values $\left(\rho_{\mathrm{w}}=2.22 \times 10^{1} \Omega \mathrm{cm}, \rho_{\mathrm{c}}=2 \times 10^{11} \Omega \mathrm{cm}, \varepsilon_{\mathrm{w}}=82\right.$ and $\left.\varepsilon_{\mathrm{c}}=8\right)$. In our previous paper [7] the effect of the assumed values on the fitted parameters was not discussed. New data and a more detailed discussion are presented below.

\subsubsection{Parameters $\varepsilon_{\mathrm{w}}$ and $\varepsilon_{\mathrm{c}}$}

The permittivities $\left(\varepsilon_{\mathrm{w}}\right.$ and $\varepsilon_{\mathrm{c}}$ ) are known with rather good precision; furthermore, it has been shown that the $\varepsilon(\xi)-\xi$ profiles are quite flat and therefore do not affect the impedance of the system in a significant way [9].

\subsubsection{Parameter $\rho_{\mathrm{c}}$}

The resistivity of the dry coating $\rho_{\mathrm{c}}$ is less well-known. Thus, recognizing that it is important to assess the magnitude of its effect, the model was regressed to the same set of data by assuming in each run a different $\rho_{\mathrm{c}}$ value, covering a rather wide range. Figure 3 shows, as an example, an experimental impedance plot, obtained with sample $\mathrm{E}$ immersed in $0.5 \mathrm{M} \mathrm{NaCl}$ after a 48-hour immersion time, compared with 7 best fitted diagrams corresponding to $\rho_{\mathrm{c}}$ values varying from $10^{10} \Omega \mathrm{cm}$ to $2 \times 10^{13} \Omega \mathrm{cm}$. In all cases, the agreement between experimental and calculated curves is good at all frequencies (with some differences at frequencies above 5 $\mathrm{kHz}$ where, as discussed above, the model is not fully satisfactory). Fig. 4 shows that the fitted parameters $\gamma$, 
$\phi(\delta)$ and $R_{\text {pore }}$ do not depend on $\rho_{\mathrm{c}}$ in a significant way, so long as $\rho_{\mathrm{c}}>5 \times 10^{10} \Omega \mathrm{cm}$. Many polymeric materials have a resistivity higher than this threshold value [14], possibly by some orders of magnitude. For example, a resistivity of $1 \times 10^{15} \Omega \mathrm{cm}$ has been reported for a dry nylon film [15]. All the results presented in the following sections were obtained by assuming $\rho_{\mathrm{c}}=2 \times 10^{11} \Omega \mathrm{cm}$, as in our previous study [7] and in other studies [16].

\subsubsection{Parameter $\rho_{\mathrm{w}}$}

The most obvious assumption about $\rho_{\mathrm{w}}$, made by ourselves in [7] and in the present study and by other authors, e.g. [11], is that it is identical to the resistivity of the electrolytic solution used in the impedance measurement. This implies that the composition of the electrolyte inside the pores should be the same as its bulk composition or, in other words that the water and the ions dissolved in it be incorporated into the coating without any selectivity. Results presented in the literature show that such an assumption might not be correct for all systems. For example, Castela and Simoes [17] mention that the diffusivity of ions is lower than that of water; Hu et al. [18] report that the diffusivity of chloride ion in epoxy coatings is ca. 3 orders of magnitude lower than the diffusivity of water; and, according to Mišković-Stanković et al. [11], the ratio between the diffusivities of water and chloride ion is slightly higher than 10. Thus, water uptake may precede ions uptake $[11,12]$, and, so long as equilibrium is not yet reached, the solution in the pores is likely to be less concentrated than the outer solution. It is also quite possible that the composition of the electrolyte within the pores would be a function of immersion time. The chemical nature of the electrolyte, especially its anion, may have important effects on the coating behavior. Duarte et al. [19], using negative-feedback scanning electrochemical microscope, have observed various topographic changes in coatings due to the water uptake and the ingress of dissolved ions depending on the electrolyte composition.

It is noteworthy that the permselective uptake of a solution less concentrated than the bulk solution would have significant consequences on (i) the determination of the $\phi(\xi)$ values and (ii) the comparison of different electrolytes. Since $\rho_{\mathrm{c}}$ is typically 8 to 10 orders of magnitude larger than $\rho_{\mathrm{w}}$, equation (1) reduces to

$$
\rho(\xi)^{-1}=\rho_{w}^{-1} \phi(\xi)
$$

already for very low $\phi(\xi)$ values. If an incorrectly low $\rho_{\mathrm{w}}$ is assumed, the water uptake is underestimated by the same factor, because larger amounts of less concentrated electrolytic solution are required to achieve a fixed $\rho(\xi)$ value. The permselectivity effects may be more or less marked for different salts and, thus, may affect the estimated water uptake to a different extent. Mišković-Stanković et al.[12] reported that the water diffusivity in epoxy coatings did not depend on nature of the anion (chloride, sulfate, naphtol-3,6-disulphonate). Since these anions have themselves different diffusivities, different electrolyte concentrations should be found in the 
pores of coatings immersed in electrolytes of the same molarity, but with different anions. In turn, different electrolyte concentrations would cause different resistivity profiles and different impedance responses.

\subsection{Electrolyte in short and through pores}

The volume fraction of the electrolyte within the through pores, henceforth denoted $\phi_{\text {pore, }}$ was determined for different coating/electrolyte combinations and various immersion times using equation (7) and the fitted $R_{\text {pore }}$ values. Table 1 reports, as an example, data relevant to coating $\mathrm{E}$ in $0.5 \mathrm{M} \mathrm{NaCl}$. The same table reports also the fitted parameters $\phi(\delta), \gamma$, and $R_{\text {pore }}$ and the volume fraction of the electrolyte in the short pores, averaged along the coating thickness $\delta$, henceforth denoted $\phi_{\mathrm{av}}$. It must be emphasized that, if $\rho_{\mathrm{w}}$ is underestimated, for the reasons discussed in the previous section, both $\phi_{\text {pore }}$ and $\phi_{\mathrm{av}}$ will be underestimated by the same factor, and their ratio will be correctly assessed. Inspection of Table 1 shows that, for all immersion times, $\phi_{\mathrm{av}}>\phi_{\text {pore, }}$ and therefore most of the electrolyte is contained in pores that do not reach the metal. However, the $\phi_{\text {pore }} / \phi_{\mathrm{av}}$ ratio increases as the immersion time increases. This suggests that, for this system, as the immersion time is prolonged and the water uptake progresses, (i) the number and/or diameter of the pores increases and (ii) the pore depth increases, such that a number of short pores become through pores at longer immersion time. All these conclusions agree with those drawn by Mišković-Stanković et al. [11] from the analysis of results of liquid sorption and thermogravimetric experiments.

It is worth pointing out that the calculation was made for cylindrical pores, perpendicular to the interfaces, which allows the volume ratio in equation (7) to be replaced by the surface area ratio. Clearly, real pores must be tortuous. Considering their tortuosity would affect $\phi_{\text {pore }}$ (because a higher tortuosity would correspond to a higher apparent $\delta$ and therefore a higher $\phi_{\text {pore }}$ ), but not $\phi_{\text {av }}$ (because Eq. (1) and (2) hold for any pore geometry). Unless tortuosity values are much higher than a few units, the conclusion that $\phi_{\mathrm{av}}>\phi_{\text {pore }}$ still holds. Once $\phi_{\text {pore }}$ is known, resistivity profiles taking into account the electrolyte in all pores may be calculated according to equation (1), in which $\phi(\xi)$ is replaced by $\phi^{\prime}(\xi)$ defined as

$$
\phi^{\prime}(\xi)=\phi_{\text {pore }}+\frac{\phi(\delta) \xi^{\gamma}}{1+\phi(\delta)\left(\xi^{\gamma}-1\right)}
$$

Fig. 5 compares these new $\rho(\xi)-\xi$ profiles (empty symbols) with those reported in Fig. 10 of reference [7] (full symbols), which were calculated by directly using the fitted $\phi(\delta)$ and $\gamma$ values and neglecting the electrolyte in the through pores. Clearly, taking $\phi_{\text {pore }}$ into account affects $\phi(\delta)$ only marginally, thus the lower resistivity limit $\rho(\delta)$ is essentially identical in this new calculation and in the previous one. Instead, for each immersion time, 
the resistivity at $\xi=0$ calculated according to equations (1) and (9) and based on $\phi^{\prime}(\xi)$ is markedly lower than that calculated according to equations (1) and (5) and based on $\phi(\xi)$.

Additional differences caused by using $\phi^{\prime}(\xi)$ instead of $\phi(\xi)$ are: (i) a more extended region where $\rho(\xi)$ is independent of $\xi$, next to the coating/oxide interface, and (ii) a progressive decrease of $\rho(0)$ with increasing immersion time. The presence of inner and outer layers in organic films was experimentally shown by Kittel et al. [20] who highlighted the heterogeneity of the properties of coatings along their thickness.

Inspection of Table 1 shows that the total water uptake (averaged along the coating thickness), given by $\phi_{\mathrm{av}}+$ $\phi_{\text {pore, }}$ remains below $10^{-4}$, i.e. below $0.01 \%$, even after $168 \mathrm{~h}$ of immersion. Such a value is significantly lower than values often reported in the literature for various coatings materials (typically between 0.1 and $10 \%$, see e.g. $[11-13,19-22])$.

Direct and reliable gravimetric data for our samples are not available. In previous sections we discussed reasons why the water uptake data in table 1 may be significantly underestimated. However, it must be stressed that, although the results of the fitting procedure are reported in part in terms of water uptake, e.g. $\phi(\delta)$, what really matters is the conductivity, given by equation (1), and, with very good approximation by equation (8). In the development of the power-law model (referred to electrolyte-penetrated coatings), $\phi(\xi)$ was assumed to be distributed along the thickness according to a power law, and the corresponding resistivity and permittivity distributions were calculated accordingly. It must be realized that $\phi(\xi)$ is just an intermediate in the calculation. The model might be directly described in terms of $\rho(\xi)$ and $\varepsilon(\xi)$, both assumed to undergo a power-law variation, over a large and a small (essentially negligible) range, respectively. Regression would then directly provide $\rho(\delta)$ and $\gamma$ values, allowing the calculation of $\rho(\xi)-\xi$ profiles identical to those shown in the present paper. In other words, the determined $\rho(\xi)-\xi$ profiles, e.g. those in Fig. 5 , are reliable, whereas the $\phi(\xi)-\xi$ may be or not reliable, depending on permselectivity effects and on the presence/absence of significant amounts of polymer-bounded water.

Analyses of the water uptake based on the evolution of the coating capacitance with immersion time often lead to its overestimation, by a small factor, as compared with gravimetric methods. Since the permittivity of water does not change dramatically with the concentration of ions dissolved in it, estimates based on capacitance are intrinsically less sensitive to selective uptake of water/ions than our approach relying on resistivity variations.

\subsection{Effect of coating formulation and electrolyte composition}


The impedance of coatings $\mathrm{E}$ and $\mathrm{O}$ was measured during immersion in $0.5 \mathrm{M} \mathrm{Na}_{2} \mathrm{SO}_{4}$, during $168 \mathrm{~h}$, and analyzed using the model described in [7], with the same approach as described above for coating E exposed to $0.5 \mathrm{M} \mathrm{NaCl}$. Coating $\mathrm{O}$, less protective than coating $\mathrm{E}$, was not studied in $\mathrm{NaCl}$ solution because signs of corrosion appeared rather soon and, to account for the corrosion process, the proposed model would need to be completed by including either additional circuit elements or an appropriate analytical expression. The agreement between experiments and model was good for all immersion times, although, as discussed before, divergences appeared at frequencies higher than $1 \mathrm{kHz}$ (which were therefore not taken into account in the fitting), as shown in Fig. 6 . Tables 2 and 3 summarize the fitting results, which are compared to those for the coating $\mathrm{E} / \mathrm{NaCl}$ system in Fig. 7. Clearly, the different coating/solution combinations show significantly different time dependencies of both $R_{\text {pore }}$ and $\phi(\delta)$. For coating E, upon increasing the immersion time, $R_{\text {pore }}$ decreases in $\mathrm{NaCl}$ solution, but increases in $\mathrm{Na}_{2} \mathrm{SO}_{4}$ solution. For coating $\mathrm{O}$ in $\mathrm{Na}_{2} \mathrm{SO}_{4}$ solution, $R_{\text {pore }}$ undergoes only small variations, initially decreasing and then slightly increasing. For coating $E$, upon increasing the immersion time, $\phi(\delta)$ increases rapidly in $\mathrm{NaCl}$ solution and remains roughly constant at a much lower value in $\mathrm{Na}_{2} \mathrm{SO}_{4}$ solution. For coating $\mathrm{O}$ in $\mathrm{Na}_{2} \mathrm{SO}_{4}$ solution, $\phi(\delta)$ is initially much higher than for coating $\mathrm{E}$ and then increases slowly when immersion time increases.

These results suggest that coating $E$ is intrinsically less defective than coating $O$, hence its lower water uptake and somewhat higher $R_{\text {pore }}$ value at short immersion time. Coating $\mathrm{O}$ undergoes then only minor changes, i.e. it does not uptake much additional water, nor significant amounts of sulfate ions diffuse from the external solution to the solution in the pores. Coating E shows diverging behavior, depending on the anion in the test solution. Supposing that the amount of water uptaken by the coating is not dramatically different in the two cases [12], the $R_{\text {pore }}$ decrease and $\phi(\delta)$ decrease might actually be the result of the progressive increase in the chloride concentration in the pores (with a resulting decrease in resistivity). Such an effect would not be observed in sulfate solution, due to a much slower diffusion of this anion. The increase in $R_{\text {pore }}$ values with exposure time, observed in $\mathrm{Na}_{2} \mathrm{SO}_{4}$ solution, is not a common phenomenon, but increasing impedances have been observed in other studies $[13,23]$. To explain the experimental observations, either a further waterinduced cross-linking of the coating material or, less probably, a pore shrinking as a consequence of coating swelling may be hypothesized. Independent evidence allowing to assess which phenomenon predominates is not available.

\section{Conclusions}


The "power law model", whose application to anti-corrosion coatings had been proposed in [7], has been revisited and employed to analyze new experimental data.

It has been shown that an inaccurate knowledge of the resistivity of the dry coating material $\rho_{\mathrm{c}}$ is unlikely to affect the quality of the agreement between model and experimental data, nor the values of the regressed parameters, at least as long as $\rho_{\mathrm{c}}$ is large. Instead, an erroneous assumption about the resistivity of the electrolyte $\left(\rho_{\mathrm{w}}\right)$ within the coating pores has a major impact on the estimated water uptake $\phi$. If, as it has been often reported, the diffusion of ions into the coating is slower that water diffusion, then $\rho_{\mathrm{w}}$ and $\phi$ will be respectively overestimated and underestimated, possibly by a large factor.

The effect of "through pores" and "short pores" has been taken simultaneously into account to calculate resistivity profiles.

Finally, it was shown that the evolution of the coating properties with immersion time depends both on the coating material, e.g. on its microstructure, and on the electrolyte, e.g. the ability of its anions to diffuse into the coating.

\section{Acknowledgments}

The experimental data were obtained in the framework of the SMILE project (Surface Mono Innovative Layer for Environment), with the financial support of the DGCIS (Direction Générale de la Compétitivité, de l'Industrie et des Services). N. Pébère thanks the partners of the project: Mapaero (Pamiers), Rescoll (Bordeaux), Airbus (Toulouse), Dassault (Paris), EADS IW (Suresnes), I'Electrolyse (Bordeaux), and the laboratories LCPO and US2B (Université de Bordeaux). 


\section{References}

[1] L. Beaunier, I. Epelboin, J.C. Lestrade, H. Takenouti, Etude électrochimique et par microscopie électronique a balayage du fer recouvert de peinture, Surf. Technol. 4 (1976) 237.

[2] M.E. Orazem, I. Frateur, B. Tribollet, V. Vivier, S. Marcelin, N.Pébère, A.L. Bunge, E.A. White, D.P. Riemer, M. Musiani, Dielectric Properties of Materials Showing Constant-Phase-Element (CPE) Impedance Response, J. Electrochem. Soc. 160 (2013) C215.

[3] D.M. Brasher, A.H. Kingsbury, Electrical measurements in the study of immersed paint coatings on metal. I. Comparison between capacitance and gravimetric methods of estimating water-uptake, J. Appl. Chem. 4 (1954) 62.

[4] G.J. Brug, A.L.G. van den Eeden, M. Sluyters-Rehbach, J.H. Sluyters, The analysis of electrode impedances complicated by the presence of a constant phase element, J. Electroanal. Chem., 176 (1984) 275.

[5] C. H. Hsu, F. Mansfeld, Concerning the conversion of the constant phase element parameter Y-O into a capacitance, Corrosion, 57, (2001) 747.

[6] B. Hirschorn, M.E. Orazem, B. Tribollet, V. Vivier, I. Frateur, M. Musiani, Determination of effective capacitance and film thickness from constant-phase-element parameters, Electrochim. Acta, 55 (2010) 6218.

[7] S. Amand, M. Musiani, M.E. Orazem, N. Pébère, B. Tribollet, V. Vivier, Constant-Phase-Element Behavior Caused by Inhomogeneous Water Uptake in Anti-Corrosion Coatings, Electrochim. Acta 87 (2013) 693.

[8] B. Hirschorn, M.E. Orazem, B. Tribollet, V. Vivier, I. Frateur, M. Musiani, Constant-Phase-Element Behavior Caused by Resistivity Distributions in Films: 1. Theory, J. Electrochem. Soc. 157 (2010) C452.

[9] M. Musiani, M.E. Orazem, N. Pébère, B. Tribollet, V. Vivier, Constant-Phase-Element Behavior Caused by Coupled Resistivity and Permittivity Distributions in Films, J. Electrochem. Soc. 158 (2011) C424. 
[10] B. Hirschorn, M.E. Orazem, B. Tribollet, V. Vivier, I. Frateur, M. Musiani, Constant-Phase-Element Behavior Caused by Resistivity Distributions in Films: 2. Applications, J. Electrochem. Soc. 157 (2010) C458.

[11] V.B. Mišković-Stanković, D.M. Dražić, M.J. Teodorović, Electrolyte penetration through epoxy coatings electrodeposited on steel, Corros. Sci. 37 (1995) 241.

[12] V.B. Mišković-Stanković, D.M. Dražić, Z. Kačarević-Popović, The sorption characteristics of epoxy coatings electrodeposited on steel during exposure to different corrosive agents, Corros. Sci. 38 (1996) 1513.

[13] C. Le Pen, C. Lacabanne, N. Pébère, Characterisation of water-based coatings by electrochemical impedance spectroscopy, Prog. Org. Coat. 46 (2003) 77.

[14] A. R. Blythe, Electrical Resistivity Measurements of Polymer Materials, Polymer Testing 4 (1984) 195.

[15] M.E. Baird, Nature of DC conductivity in polyamides, J. Polymer Sci. A-2 8 (1970) 739.

[16] B.R. Hinderliter, S.G. Croll, D.E. Tallman, Q. Su, G.P. Bierwagen, Interpretation of EIS data from accelerated exposure of coated metals based on modeling of coating physical properties, Electrochim. Acta 51 (2006) 4505.

[17] A.S. Castela, A.M. Simoes, Assessment of water uptake in coil coatings by capacitance measurements, Prog. Org. Coat. 46 (2003) 55.

[18] J.M. Hu, J.Q. Zhang, C.N. Cao, Determination of water uptake and diffusion of $\mathrm{Cl}^{-}$ion in epoxy primer on aluminum alloys in $\mathrm{NaCl}$ solution by electrochemical impedance spectroscopy, Prog. Org. Coat. 46 (2003) 273.

[19] R.G. Duarte, S. González, A.S. Castela, M.G.S. Ferreira, R.M. Souto, Sensing polymer inhomogeneity in coated metals during the early stages of coating degradation, Prog. Org. Coat. 74 (2012) 365.

[20] J. Kittel, N. Celati, M. Keddam, H. Takenouti, New methods for the study of organic coatings by EIS: New insights into attached and free films, Prog. Org. Coat. 41 (2001) 93.

[21] J.H. Park, G.D. Lee, H. Ooshige, A. Nishikata, T. Tsuru, Monitoring of water uptake in organic coatings under cyclic wet-dry condition, Corros. Sci. 45 (2003) 1881. 
[22] R.G. Duarte, A.S. Castela, M.G.S. Ferreira, A new model for estimation of water uptake of an organic coating by EIS: the tortuosity pore model, Prog. Org. Coat. 65 (2009) 197.

[23] M.C.S.S. Macedo, I.C.P. Margarit-Mattos, F.L. Fragata, J.-B. Jorcin, N. Pébère, O.R. Mattos, Contribution to a better understanding of different behaviour patterns observed with organic coatings evaluated by electrochemical impedance spectroscopy, Corros. Sci. 51 (2009) 1322. 
Table 1. Dependence of the fitted parameters $\phi(\delta), \gamma$ and $R_{\text {pore }}$ and of the calculated quantities $\phi_{\mathrm{av}}$ and $\phi_{\text {pore }}$ on the immersion time in $0.5 \mathrm{M} \mathrm{NaCl}$ for coating $\mathrm{E}$.

\begin{tabular}{c|c|c|c|c|c}
$\begin{array}{c}\text { Immersion } \\
\text { time in 0.5 } \\
\mathrm{M} \mathrm{NaCl} / \\
\mathrm{h}\end{array}$ & $\begin{array}{c}\phi(\delta) / \\
\text { dimensionless }\end{array}$ & $\begin{array}{c}\gamma / \\
\text { dimensionless }\end{array}$ & $\begin{array}{c}R_{\text {pore }} / \\
\Omega \mathrm{cm}^{2}\end{array}$ & $\begin{array}{c}\phi_{\text {av }} / \\
\text { dimensionless }\end{array}$ & $\begin{array}{c}\phi_{\text {pore }} / \\
\text { dimensionless }\end{array}$ \\
\hline 24 & $1.5 \times 10^{-5}$ & 3.5 & $1.0 \times 10^{6}$ & $3.2 \times 10^{-6}$ & $4.3 \times 10^{-8}$ \\
\hline 48 & $2.2 \times 10^{-5}$ & 3.4 & $3.1 \times 10^{5}$ & $5.1 \times 10^{-6}$ & $1.4 \times 10^{-7}$ \\
\hline 72 & $3.2 \times 10^{-5}$ & 3.1 & $2.1 \times 10^{5}$ & $7.8 \times 10^{-6}$ & $2.1 \times 10^{-7}$ \\
\hline 168 & $1.2 \times 10^{-4}$ & 3.0 & $5.1 \times 10^{4}$ & $2.9 \times 10^{-5}$ & $8.6 \times 10^{-7}$
\end{tabular}

Table 2. Dependence of the fitted parameters $\phi(\delta), \gamma$ and $R_{\text {pore }}$ and of the calculated quantities $\phi_{\mathrm{av}}$ and $\phi_{\mathrm{pore}}$ on the immersion time in $0.5 \mathrm{M} \mathrm{Na}_{2} \mathrm{SO}_{4}$ for coating $\mathrm{E}$.

\begin{tabular}{c|c|c|c|c|c}
$\begin{array}{c}\text { Immersion } \\
\text { time in 0.5 } \\
\mathrm{M} \mathrm{Na}_{2} \mathrm{SO}_{4} \\
/ \mathrm{h}\end{array}$ & $\begin{array}{c}\phi(\delta) / \\
\text { dimensionless }\end{array}$ & $\begin{array}{c}\gamma / \\
\text { dimensionless }\end{array}$ & $\begin{array}{c}R_{\text {pore }} / \\
\Omega \mathrm{cm}^{2}\end{array}$ & $\begin{array}{c}\phi_{\text {av }} / \\
\text { dimensionless }\end{array}$ & $\begin{array}{c}\phi_{\text {pore }} / \\
\text { dimensionless }\end{array}$ \\
\hline 6 & $2.3 \times 10^{-6}$ & 3.6 & $8.5 \times 10^{5}$ & $5.3 \times 10^{-7}$ & $3.9 \times 10^{-8}$ \\
\hline 24 & $3.9 \times 10^{-6}$ & 4.0 & $1.9 \times 10^{6}$ & $8.0 \times 10^{-7}$ & $1.8 \times 10^{-8}$ \\
\hline 48 & $1.6 \times 10^{-6}$ & 3.9 & $2.5 \times 10^{6}$ & $3.5 \times 10^{-7}$ & $1.3 \times 10^{-8}$ \\
\hline 72 & $3.8 \times 10^{-6}$ & 4.2 & $2.9 \times 10^{6}$ & $7.9 \times 10^{-7}$ & $1.1 \times 10^{-8}$ \\
\hline 168 & $2.2 \times 10^{-6}$ & 4.2 & $2.9 \times 10^{6}$ & $4.4 \times 10^{-7}$ & $1.1 \times 10^{-8}$ \\
& & & & &
\end{tabular}


Table 3. Dependence of the fitted parameters $\phi(\delta), \gamma$ and $R_{\text {pore }}$ and of the calculated quantities $\phi_{\mathrm{av}}$ and $\phi_{\text {pore }}$ on the immersion time in $0.5 \mathrm{M} \mathrm{Na}_{2} \mathrm{SO}_{4}$ for coating $\mathrm{O}$.

\begin{tabular}{c|c|c|c|c|c} 
Immersion & $\phi(\delta) /$ & $\gamma /$ & $R_{\text {pore }} /$ & $\phi_{\text {av }} /$ & $\phi_{\text {pore }} /$ \\
time in 0.5 & dimensionless & dimensionless & $\Omega \mathrm{cm}^{2}$ & dimensionless & dimensionless \\
$\mathrm{M} \mathrm{Na}_{2} \mathrm{SO}_{4}$ & & & & \\
\hline 6 & $3.7 \times 10^{-5}$ & 3.9 & $6.7 \times 10^{5}$ & $7.7 \times 10^{-6}$ & $4.9 \times 10^{-8}$ \\
\hline 6 & $2.8 \times 10^{-5}$ & 3.4 & $6.7 \times 10^{5}$ & $6.5 \times 10^{-6}$ & $5.0 \times 10^{-8}$ \\
\hline 24 & $4.3 \times 10^{-5}$ & 3.4 & $5.4 \times 10^{5}$ & $9.8 \times 10^{-6}$ & $6.2 \times 10^{-8}$ \\
\hline 120 & $4.4 \times 10^{-5}$ & 3.2 & $7.8 \times 10^{5}$ & $1.0 \times 10^{-5}$ & $4.3 \times 10^{-8}$ \\
\hline 144 & $5.2 \times 10^{-5}$ & 3.3 & $8.3 \times 10^{5}$ & $1.2 \times 10^{-5}$ & $4.0 \times 10^{-8}$ \\
\hline 168 & $5.3 \times 10^{-5}$ & 3.3 & $9.5 \times 10^{5}$ & $1.2 \times 10^{-5}$ & $3.5 \times 10^{-8}$ \\
\hline & & & & &
\end{tabular}




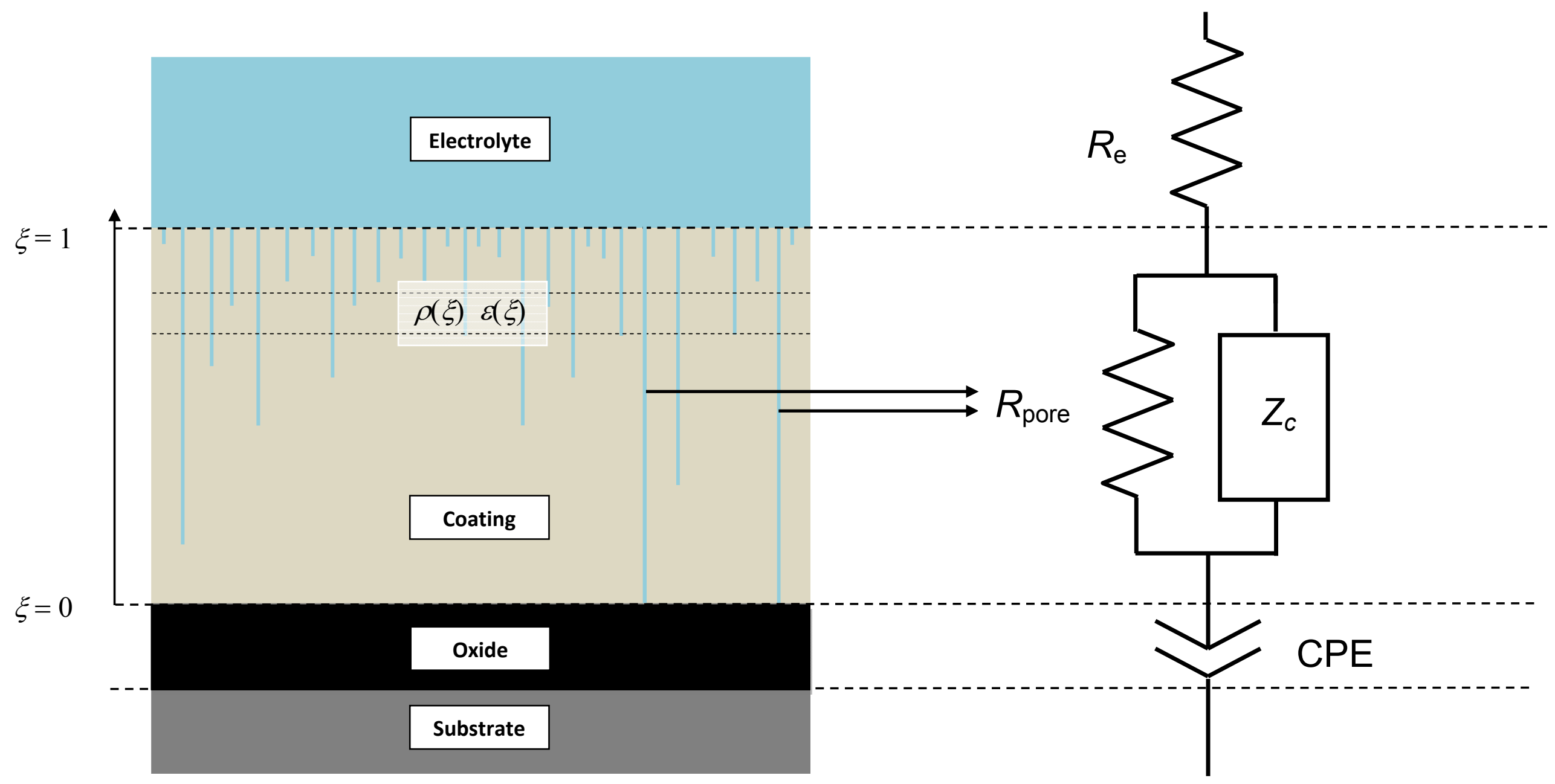

Figure 1. Schematic representation of a substrate/oxide/coating/electrolyte system. $R_{e}$ is the uncompensated electrolyte resistance; $R_{p o r e}$ accounts for the through pores joining the coating/electrolyte and coating/oxide interface; $Z_{c}$ is the impedance of the electrolyte-penetrated coating described by Eq. (3); the CPE represents the oxide layer covering the Al 2024 alloy. 


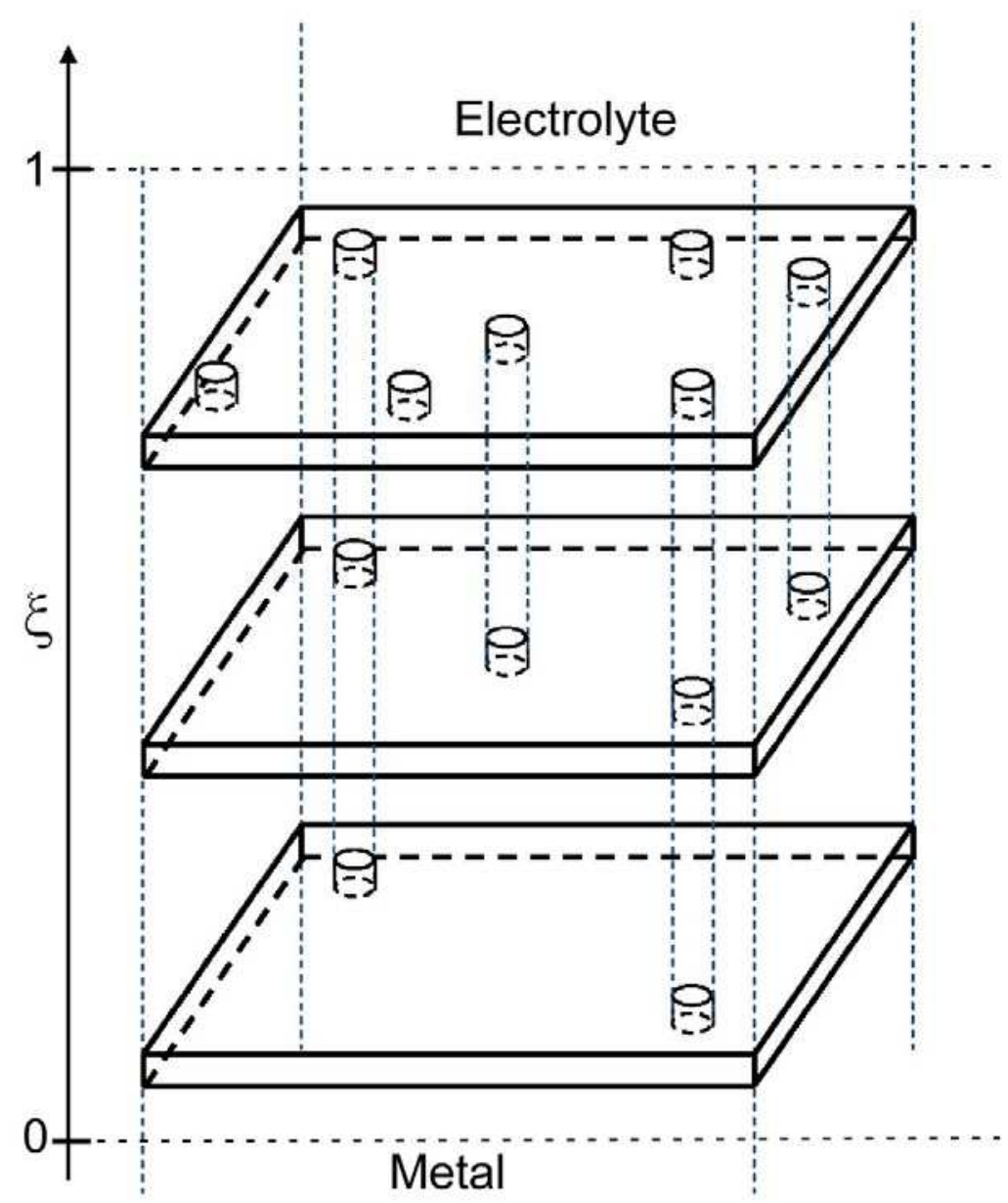

Figure 2. Schematic representation of thin layers of the coating material located at different $\xi$ positions, highlighting that the number of pores decreases as $\xi$ becomes smaller, i.e. as the distance from the coating/electrolyte interface increases. 

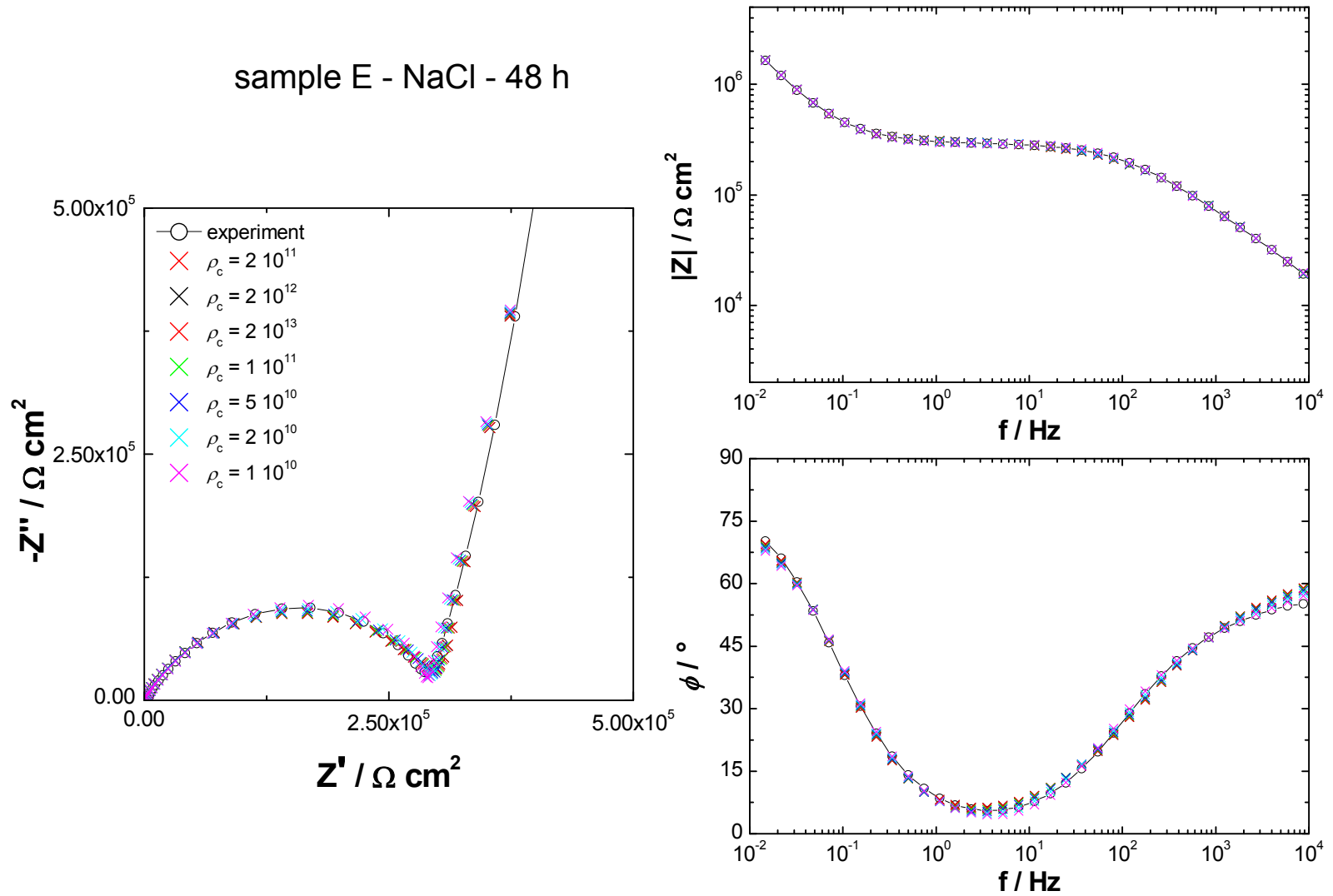

Figure 3. Comparison of the experimental impedance plots obtained with sample E exposed to $0.5 \mathrm{M} \mathrm{NaCl}$, after a 48-hour immersion time, with the best fitted curves corresponding to the $\rho_{\mathrm{c}}$ values indicated on the Nyquist plot. 

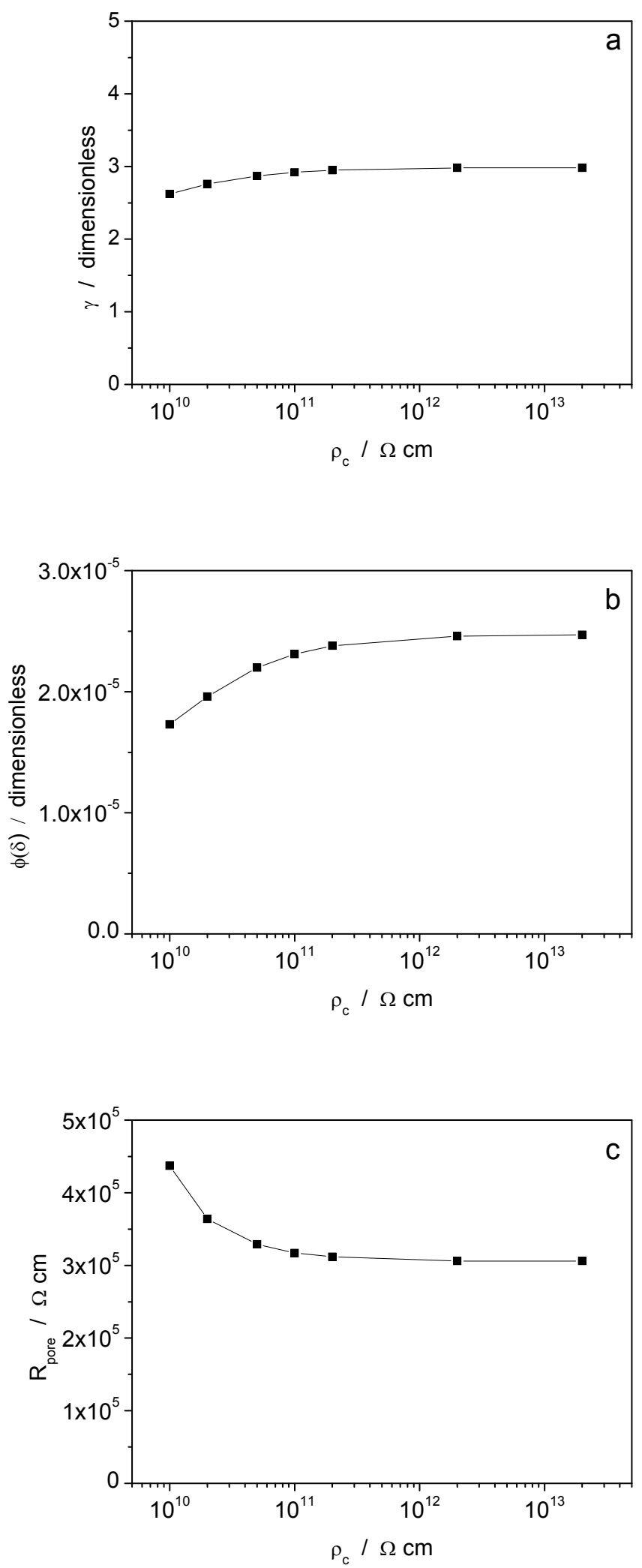

Figure 4. Effect of the assumed $\rho_{\mathrm{c}}$ value on the best fitted parameters $\gamma(\mathrm{a}), \phi(\delta)$ (b) and $R_{\text {pore }}(\mathrm{c})$. 


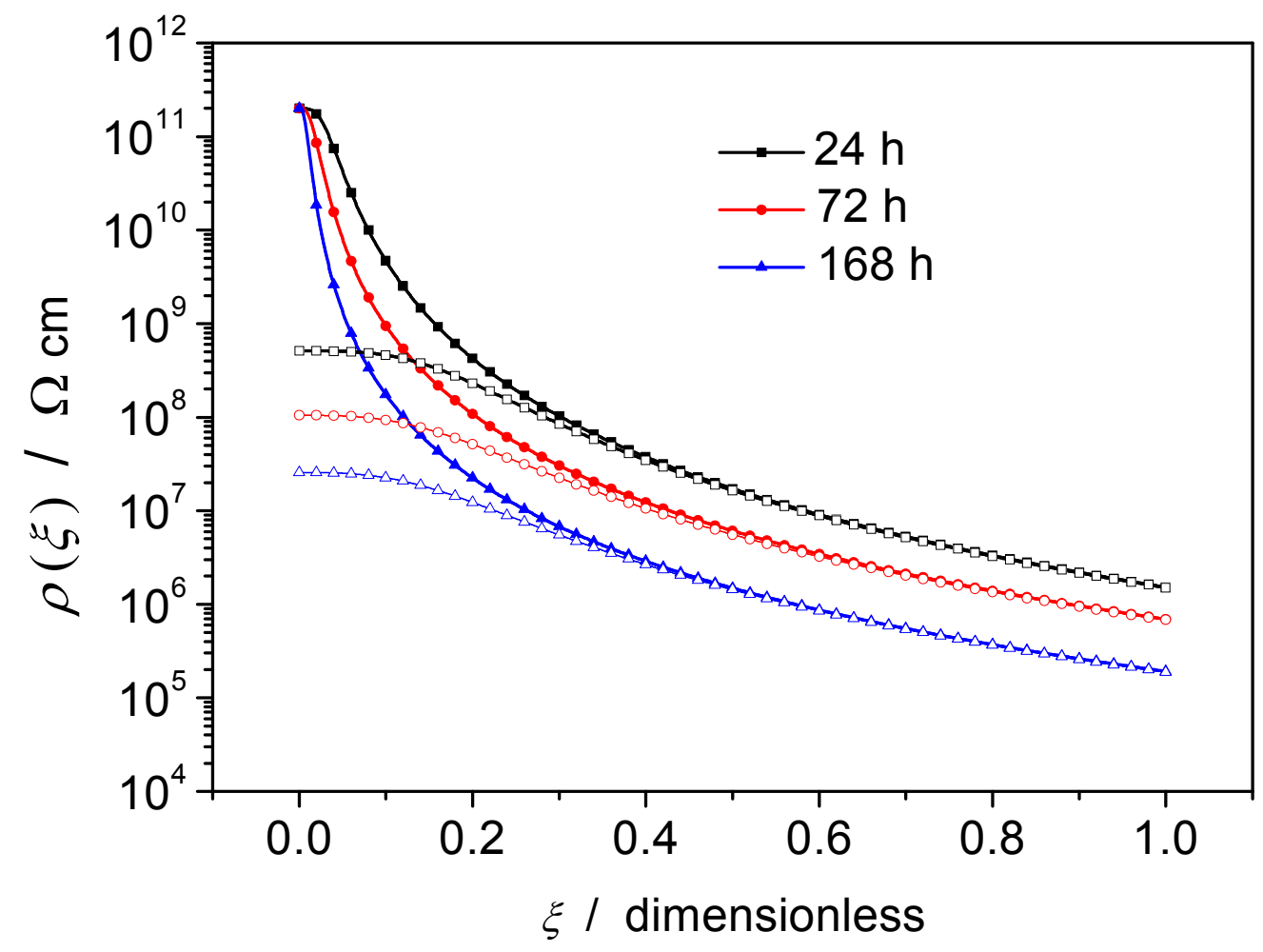

Figure 5. Comparison of the resistivity profiles calculated for the Coating $\mathrm{E} / \mathrm{NaCl}$ solution system, calculated on the basis of total water uptake (empty symbols) or water uptake in short pores only (full symbols) Immersion times are indicated on the figure. 

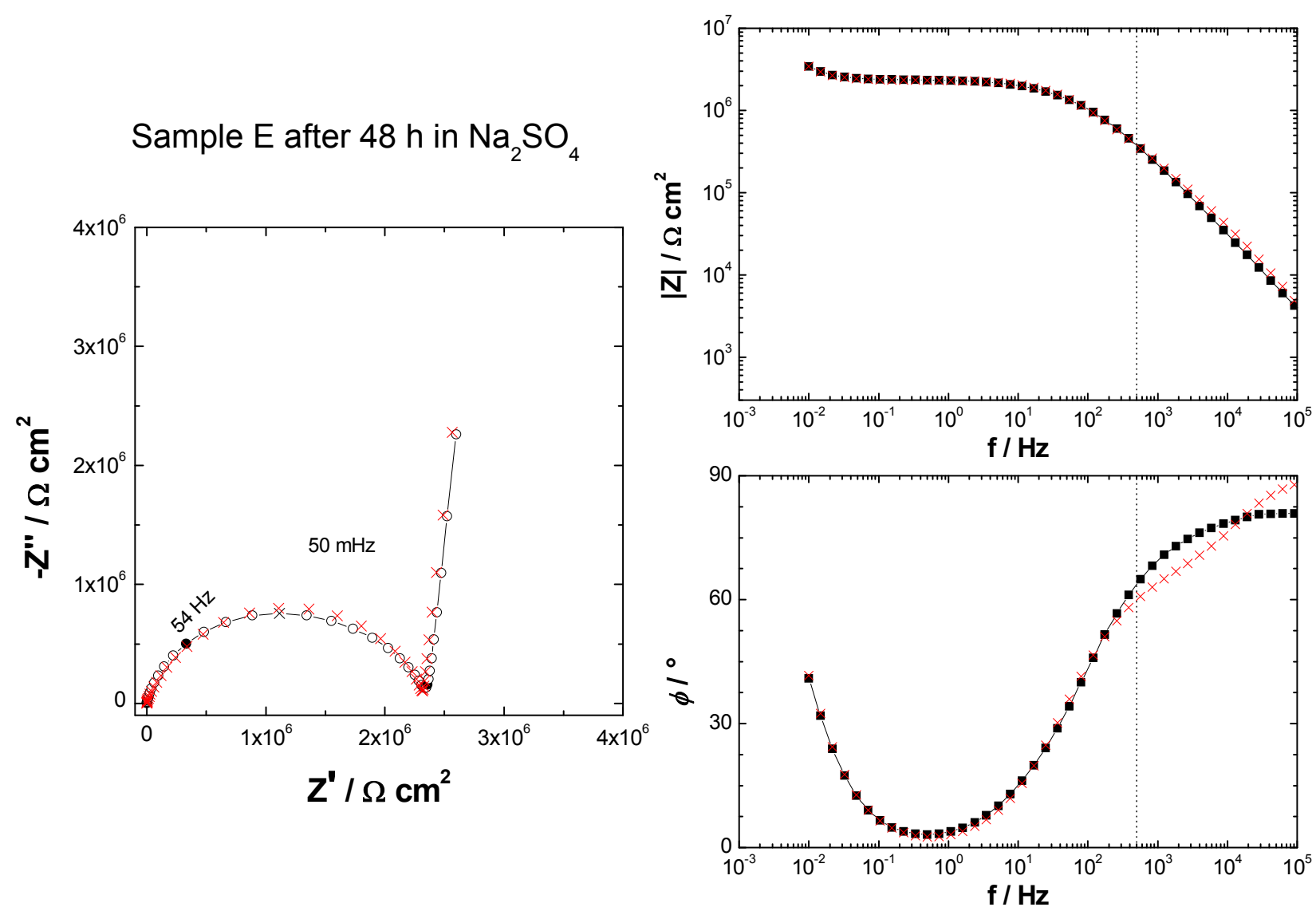

Figure 6. Comparison of the experimental impedance plots obtained with sample E exposed to $0.5 \mathrm{M}$ $\mathrm{Na}_{2} \mathrm{SO}_{4}$, after a 48-hour immersion time, with the best fitted curve obtained by assuming $\rho_{\mathrm{w}}=22.2 \Omega \mathrm{cm}, \rho_{\mathrm{c}}$ $=2 \times 10^{11} \Omega \mathrm{cm}, \varepsilon_{\mathrm{w}}=82$ and $\varepsilon_{\mathrm{c}}=8$. 

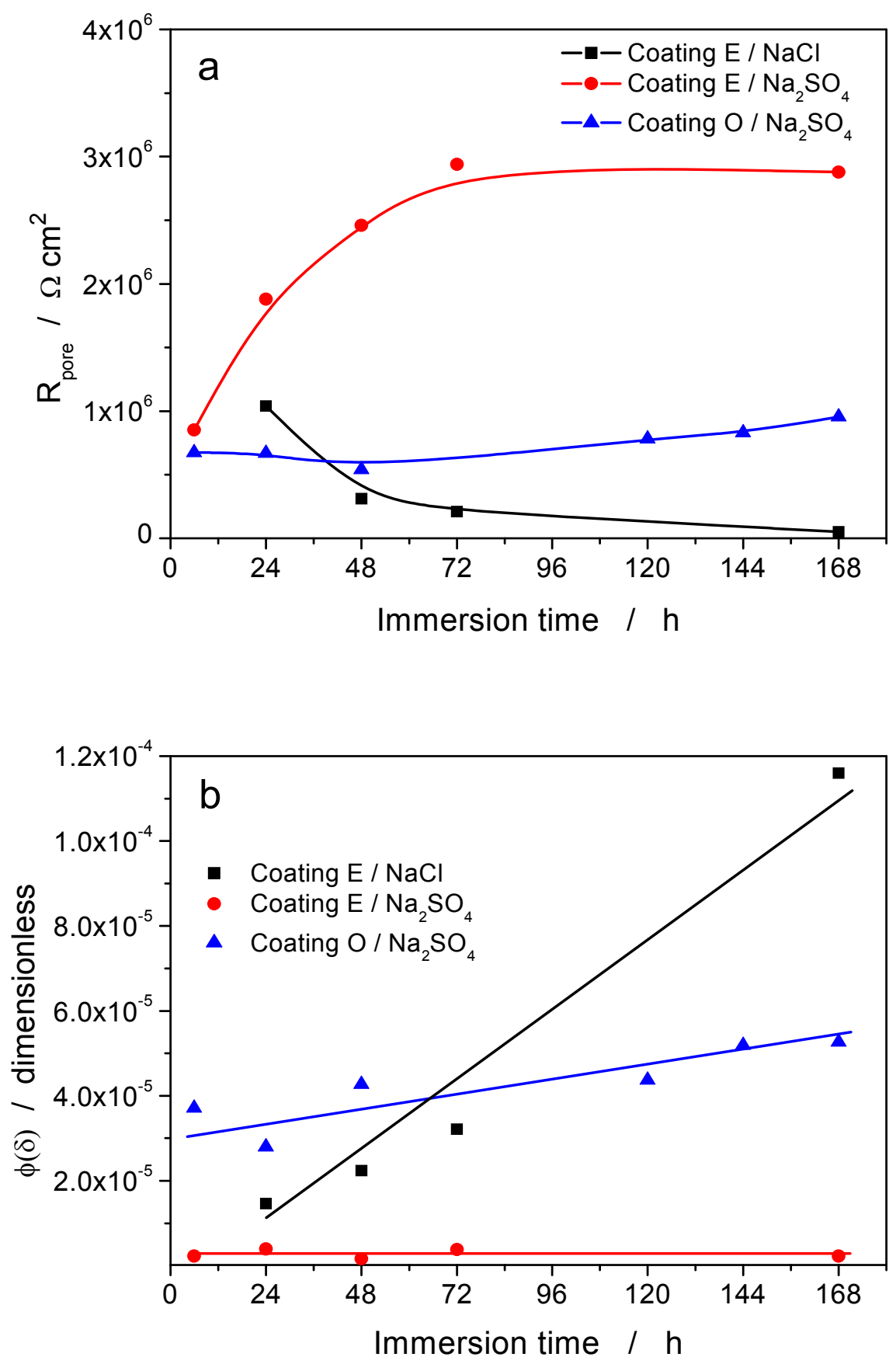

Figure 7. Dependence of $R_{\text {pore }}$ and $\phi(\delta)$ on the immersion time for the coating/solution combinations indicated on the figure. The lines are only an aid for the eye. 